BNL - 72150-2004-CP

UCLA - 03C

\title{
Progress in Designing a Muon Cooling Ring with Lithium Lenses
}

\author{
Y. Fukui, David B. Cline, Alper A. Garren \\ Univ. of California at Los Angeles \\ and \\ Harold G. Kirk \\ Brookhaven National Laboratory
}

\begin{abstract}
This work was supported by the U.S. Department of Energy under Contract DE-ACO2-98CH10886.
\end{abstract}

November 2003

Proc. of the Particle Accelerator Conference (PAC2003), Portland, OR, May 12-16, 2003, pg. 1787-89, on $C D$ (TPPB084). 


\title{
PROGRESS IN DESIGNING A MUON COOLING RING WITH LITHIUM LENSES
}

\author{
Y. Fukui*, David B. Cline, Alper A. Garren, University of California at Los Angeles, CA \\ Harold G. Kirk, Brookhaven National Laboratory, NY
}

\begin{abstract}
We discuss particle tracking simulations in a storage ring with lithium lens inserts designed for the six-dimensional phase space cooling of muons by the ionization cooling. The ring design contains one or more lithium lens absorbers for transverse cooling that transmit the beam with very smail beta-function values, in addition to liquidhydrogen wedge-shaped absorbers in dispersive locations for longitudinal cooling. Such a ring could comprise the final component of a cooling system for use in a muon collider. The beam matching between dipole-quadrupole lattices and the lithium lenses is of particular interest.
\end{abstract}

\section{OVERVIEW}

The ionization cooling is one of the most promising method to reduce the 6 dimensional phase space of muon beam, where both transverse and longitudinal momenta are reduced due to the energy loss in absorbers, and only longitudinal components of the muon momenta are restored through the accelerating fields of RF cavities. The multiple Coulomb scattering contributes to heat the transverse phase space. And the $\mathrm{dE} / \mathrm{dx}$ straggling through absorbers contributes to heat the longitudinal phase space. Wedge absorbers in dispersive region in bending cells perform the emittance exchange between the longitudinal emittance and the horizontal emittance. [1]

Lithium lens is an active focusing element with energy absorber function at the same time. With $\beta$ at $1 \mathrm{~cm}$ with high current density Lithium lenses, the equilibrium normalized transverse emittance can be at around $100 \mathrm{~mm}$. mrad, which is necessary for a $\mu^{+} \mu^{-}$collider. [2]

In the muon cooling ring with Lithium lenses, Lithium lenses with the $\beta$ function as low as $1.0 \mathrm{~cm}$ is placed in a straight section with matching solenoid magnets. Figure 1 shows a schematic view of a muon cooling ring with a Lithium lens. Circumference is $37.5 \mathrm{~m}$, the straight section is $5.9 \mathrm{~m}$ long each, and the radius of the bending section is $4.6 \mathrm{~m}$.

\section{MUON COOLING IN STRAIGHT CHANNELS WITH LITHIUM LENSES}

We designed muon cooling rings with a Lithium lens which is made of 2 matching higher $\beta$ Lithium lenses sandwiching the central lower $\beta$ Lithium lens. $\beta$ at the inner 22 $\mathrm{cm}$ long Lithium lens is $1.0 \mathrm{~cm}$. The matching Lithium rod with the length of $6.3 \mathrm{~cm}$ each, which sandwich the central

\footnotetext{
*fukui@slac.stanford.edu
}

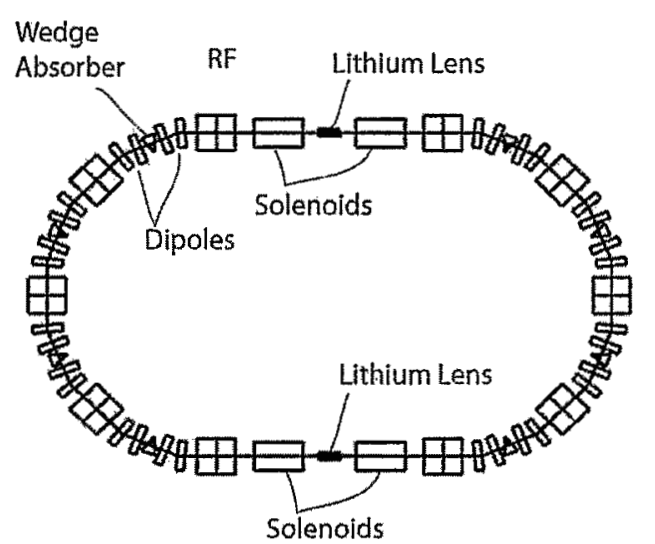

Figure 1: A schematic diagram of a muon cooling ring with Lithium lenses in straight sections.

Lithium lens, has an equilibrium $\beta$ at $4.0 \mathrm{~cm}$, which swings the $\beta$ function from the $\beta$ at $16 \mathrm{~cm}$ at the outer end to the $\beta$ at $1 \mathrm{~cm}$ at the inner end of the matching Lithium rod. The solenoids has 6 Tesla $B_{z}$ field where the $B_{z}$ direction of solenoids is opposite to each other, and each solenoid is $1.3 \mathrm{~m}$ long Figure 2 shows a schematic diagram of a Lithium lens and straight section which is made of 2 matching solenoids and a set of Lithium lenses. Figure 3 shows the $\beta$ as a function of $\mathrm{z}$ in the Lithium lens and matching cells with solenoids.

In order to study the muon beam dynamics through a Lithium lens and matching solenoid lattices which sandwich the Lithium lens, we performed tracking simulation with ICOOL tracking code. [3] Original model was designed by using the SYNCH [4] which generates the input date for the tracking code ICOOL.

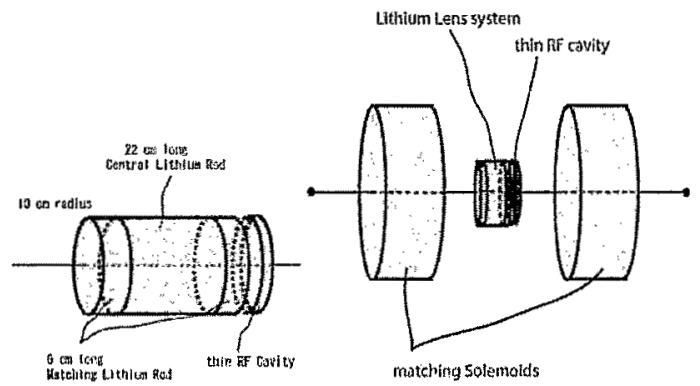

Figure 2: A schematic diagram of a Lithium lens(left) and straight section(right).

Figure 4 shows the development of the normalized transverse emittance as a function of $\mathrm{z}$ through 33 sets of $5.9 \mathrm{~m}$ 


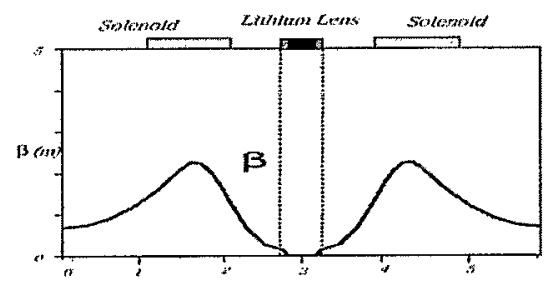

Figure 3: $\beta$ as a function of $s$ in the Lithium lens and matching cells with solenoids.

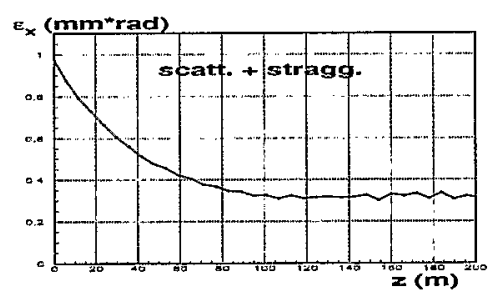

Figure 4: Normalized transverse emittance as a function of $\mathrm{z}$ through 33 sets of straight sections.

long straight channel. In this simulation, the loss of muon $p_{z}$ due to the $\mathrm{dE} / \mathrm{dx}$ energy loss through the Lithium lens is recovered through a thin RF cavity by adding average $p_{z}$ kick. The equilibrium normalized transverse emittance is around $0.3 \mathrm{~mm} \cdot \mathrm{rad}$. Figure 5 and 6 show the development of the normalized longitudinal emittance, $\Delta p / p$ and $\Delta z$ as a function of $\mathrm{z}$, and the transmission as a function of $z$, respectively.

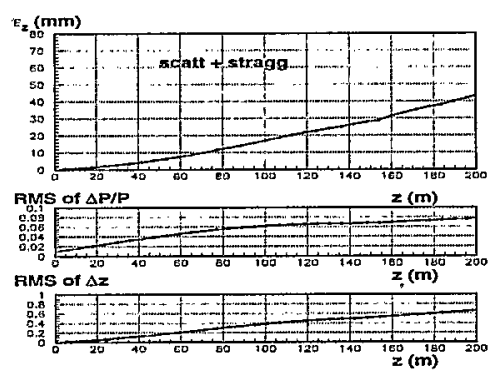

Figure 5: Normalized longitudinal emittance, $\Delta p / p$ and $\Delta z$ as a function of $\mathrm{z}$ through 33 sets of straight sections.

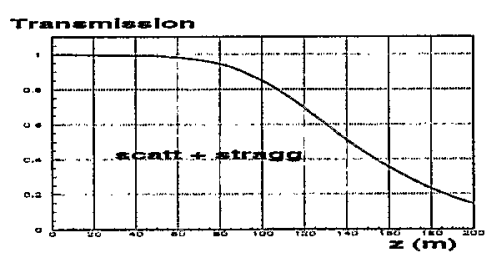

Figure 6: Muon transmission as a function of $\mathrm{z}$ through 33 sets of straight sections.

\section{MUON COOLING IN A COOLING RING WITH LITHIUM LENSES}

We designed a 45 degree bending cell by using two sets of zero-gradient dipole magnets with edge focusing. Wedge absorbers of liq. $\mathrm{H}_{2}$ are placed in dispersive regions in the bending cells. RF cavities are placed wherever the space is available.

Figure 7 shows $\beta$ vs. $\mathrm{z}$ and $D$ vs. $\mathrm{z}$ in a muon cooling ring with Lithium lenses in straight sections. The $\beta$ vs. $\mathrm{z}$ and $D$ vs. $\mathrm{z}$ in a 45 degree bending cell is shown in Figure 8 We placed $1.7 \mathrm{~cm}$ long liq. $\mathrm{H}_{2}$ wedge absorbers at the center of dispersive regions in the bending cells.

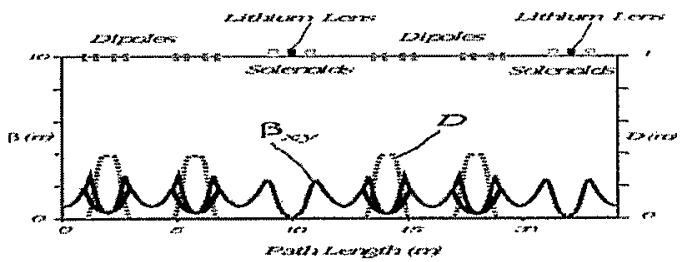

Figure 7: $\beta$ vs. $\mathrm{z}$ and $D$ vs. $\mathrm{z}$ in a muon cooling ring with Lithium lenses in straight sections

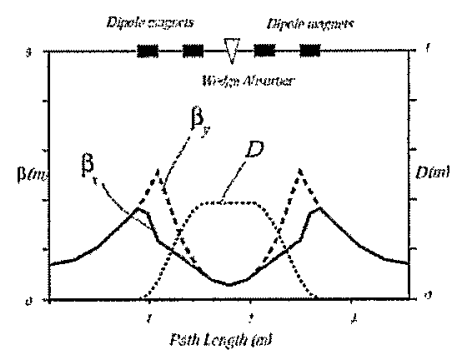

Figure 8: $\beta$ vs. $\mathrm{z}$ and $D$ vs. $\mathrm{z}$ in a 45 degree bending cell

Table 1 lists parameters of the muon cooling ring with two Lithium lenses in straight channels.

Table 1: Parameters of a muon cooling ring

\begin{tabular}{ll}
\hline \hline muon momentum & $250 \mathrm{MeV} / \mathrm{c}$ \\
Circumference & $37.5 \mathrm{~m}$ \\
straight section length & $5.9 \mathrm{~m}(\times 2)$ \\
Structure of half cell & 2 dipoles with edges \\
number of bending cells & 8 \\
bend cell length & $3.6 \mathrm{~m}$ \\
length of Lithium lens & $34.5 \mathrm{~cm}(\times 2)$ \\
lowest $\beta$ in Lithium lens & $1.0 \mathrm{~cm}$ \\
energy loss & $35 \mathrm{MeV} /$ turn \\
dipole bend angles & $44.2,-21.7$ degree \\
dipole edge angles & $30 /-3,-11 /-11$ degree \\
dipole magnetic field & $6.5,-3.2$ tesla \\
Cell tunes bend cell & $0.72 / 0.70$ \\
Cell tunes straight cell & 4.0 \\
\hline \hline
\end{tabular}


Figure 9 shows the development of the normalized transverse emittance as a function of $\mathrm{z}$ through 8 turns of the muon cooling ring in Figure 1. channel. In this simulation, the loss of muon $p_{z}$ due to the $\mathrm{dE} / \mathrm{dx}$ energy loss through the Lithium lens or Liq. $\mathrm{H}_{2}$ wedge absorbers is recovered through a thin RF cavity by adding average $p_{z}$ kick. The figure indicates the transverse cooling in the muon cooling ring with Lithium lenses. Figure 10 and 11 show the development of the normalized longitudinal emittance, $\Delta p / p$ and $\Delta z$ as a function of $z$, and the transmission as a function of $\mathrm{z}$, respectively.

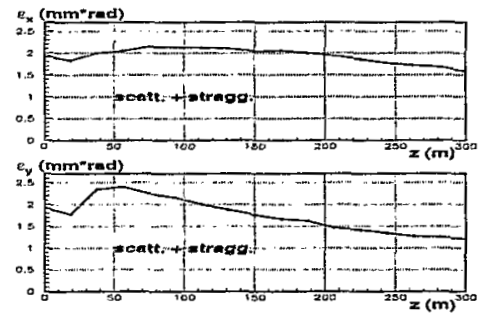

Figure 9: Normalized transverse emittance as a function of $\mathrm{z}$ through 8 turns of a muon cooling ring.

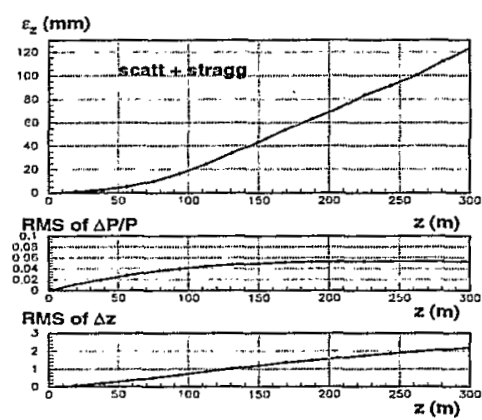

Figure 10: Normalized longitudinal emittance, $\Delta p / p$ and $\Delta z$ as a function of $\mathrm{z}$ through 8 turns of a muon cooling ring.

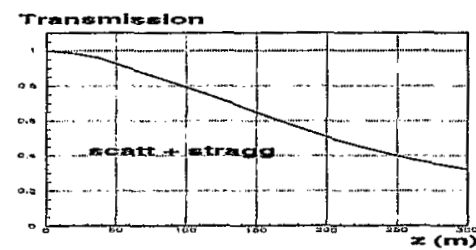

Figure 11: Muon transmission as a function of $z$ through 8 turns of a muon cooling ring.

\section{CONCLUSION}

We designed a race track muon cooling ring with $35 \mathrm{~cm}$ long Lithium lenses in straight channels with $\beta$ at $1 \mathrm{~cm}$. Bending cells have zero-gradient dipole magnets with edge focusing, and wedge absorbers in dispersive regions. Study is in progress to obtain the 6 dimensional muon cooling in this cooling ring.

\section{ACKNOWLEDGMENTS}

The authors wish to thank R. B. Palmer, R. Fernow, A Sessler, M. Zisman, J. Wurtele, D. Neuffer, and V. Balbekov, for useful discussions, and their help and encouragement.

\section{REFERENCES}

[1] H. G. Kirk, D. B. Cline, Y. Fukui, A. A. Garren, Progress Toward A Muon Ring Cooler, SNOWMASS-2001-M101, Jun 2001. 5pp., Prepared for APS / DPF / DPB Summer Study on the Future of Particle Physics (Snowmass 2001), Snowmass, Colorado, 30 Jun - 21 Jui 2001.

[2] C. M. Ankenbrandt, et., al, the NuMu Collaboration, Status of Neutrino Factory and Muon Collider Reasearch and Developmemt and Future Plans, Phys. rev. ST Accel. Beams 2 (1999) 081001

[3] R. Fernow, A Simulation Code for Ionization Cooling of Muon Beams, Part. Accel. Conf., Edts., A. Luccio and W. MacKay, Proc. 1999, p. 3020.

[4] A. A. Garren, A. S. Kenney, E. D. Courant, A. D. Russel, and M. J. Syphers. SYNCH - A Compueter System for Synchrotron Design and Orbit Analysis, User's Guide, SSCLMAN-0030, 1993 